\title{
Factors influencing the performance of a diagnostic model including contrast-enhanced ultrasound in 1023 breast lesions: comparison with histopathology
}

\author{
Yijie Chen ${ }^{1 \#}$, Lina Tang ${ }^{1 \#}$, Zhongshi Du ${ }^{1}$, Zhaoming Zhong ${ }^{1}$, Jun Luo ${ }^{2}$, Lichun Yang ${ }^{3}$, Ruoxia Shen ${ }^{3}$, \\ Yan Cheng ${ }^{4}$, Zizhen Zhang ${ }^{4}$, Ehui Han ${ }^{5}$, Zhihong Lv ${ }^{5}$, Lijun Yuan ${ }^{6}$, Yong Yang ${ }^{6}$, Yinrong Cheng ${ }^{7}$, \\ Lei Yang ${ }^{7}$, Shengli Wang ${ }^{8}$, Baoyan Bai ${ }^{8}$, Qin Chen ${ }^{2}$
}

${ }^{1}$ Department of Ultrasound, Fujian Cancer Hospital \& Fujian Medical University Cancer Hospital, Fuzhou 350014, China; ${ }^{2}$ Department of Ultrasound, Sichuan Provincial People's Hospital, Chengdu 610072, China; ${ }^{3}$ Department of Ultrasound, the Third Affiliated Hospital of Kunming Medical University \& Yunnan Cancer Hospital, Kunming 650118, China; ${ }^{4}$ Department of Ultrasound, Qujing City First People's Hospital, Qujing 655000, China; ${ }^{5}$ Department of Ultrasound, Huangshi Central Hospital, Affiliated Hospital of Hubei Polytechnic University, Edong Healthcare Group, Huangshi 435000, China; ${ }^{6}$ Departments of Ultrasound, Tangdu Hospital, Fourth Military Medical University, Xi'an 710032, China; ${ }^{7}$ Department of Ultrasound, Chengdu First People's Hospital, Chengdu 610000, China; ${ }^{8}$ Department of Ultrasound, Yanan University Affiliated Hospital, Yan'an 716000, China

Contributions: (I) Conception and design: L Tang; (II) Administrative support: Q Chen; (III) Provision of study materials or patients: All authors; (IV) Collection and assembly of data: Y Chen; (V) Data analysis and interpretation: Y Chen; (VI) Manuscript writing: All authors; (VII) Final approval of manuscript: All authors.

\#These authors contributed equally to this work.

Correspondence to: Lina Tang. Department of Ultrasound, Fujian Cancer Hospital \& Fujian Medical University Cancer Hospital, 420 Fuma Road, Fuzhou 350014, China. Email: tanglina@fjzlhospital.com; Qin Chen. Department of Ultrasound, Sichuan Provincial People’s Hospital, 32 First Ring Road, Qingyang District, Chengdu 610072, China. Email: 1718686103@qq.com.

Background: We aimed to investigate the influence of patient and lesion characteristics on our diagnostic model for contrast-enhanced ultrasound (CEUS) of the breast, comparing its accuracy with that of histopathology.

Methods: Conducting a study with eight medical centers, we compared 1,023 breast lesions categorized as BI-RADS 4 or 5 with the score from our newly-established CEUS-based diagnostic model, comparing the results with pathological outcomes. Univariate and multivariate logistic regression analyses were conducted to determine the influence of clinicopathological characteristics on the performance of this CEUS model.

Results: Logistic regression analysis showed that patients' age, maximum lesion diameter, and distance from the lesion's deep edge to the pectoralis major were significant independent influencing factors. The model's diagnostic accuracy was greater for patients $>35 \mathrm{y}(\mathrm{P}=0.005)$, for maximum lesion diameter $>20 \mathrm{~mm}$, and for distance from the lesion's deep edge to the pectoralis major $\leq 3.05 \mathrm{~mm}$. There was no significant difference in accuracy between lesions with maximum lesion diameter 10-20 and <10 mm (P=0.393).

Conclusions: The diagnostic performance of the proposed CEUS model for breast lesions is influenced by patients' age, maximum lesion diameter, and distance from the lesion's deep edge to the pectoralis major. Consideration of influencing factors is required to optimize clinical use of the CEUS model.

Keywords: Breast cancer; ultrasonography; contrast-enhanced ultrasound

Submitted Jun 23, 2019. Accepted for publication Oct 10, 2019.

doi: $10.21037 /$ atm.2019.10.83

View this article at: http://dx.doi.org/10.21037/atm.2019.10.83 


\section{Introduction}

Breast cancer is the most commonly diagnosed cancer and is also the leading cause of cancer death in the vast majority of countries among women. Worldwide, there were about 2.1 million newly diagnosed female breast cancer cases in 2018, accounting for almost 1 in 4 cancer cases (1). The clinical management of patients with breast lesions is complex. Early diagnosis of breast lesions allows for earlier treatment and better prognosis (2). Mammography and ultrasonography are proven methods for breast cancer screening and diagnosis (3). For Asian women, who characteristically have relatively thin and higher-density breasts, it must be taken into account that the accuracy of mammography images interpretation is reduced in this patient population $(3,4)$.

Recognizing the widespread use of ultrasonography (US) in breast imaging, the American College of Radiology (ACR) published the fourth edition of the Breast Imaging Reporting and Data System (BI-RADS) lexicon for US in 2003 (5). Lesions categorized as BI-RADS category 4 or 5 are usually directed to biopsy using ultrasound-guided fineneedle aspiration (FNA) or core biopsy $(6,7)$, although the positive rate is only $17 \%$ in Category 4 (8). In other words, it comes with a substantial risk of false positives (i.e. biopsy with benign results).

The US lexicon mainly includes morphologic findings and associated features that also include vascularity with the subcategories absent vascularity, internal vascularity, and vessels in rim, but lacks microcirculation information of solid breast masses. Although breast cancer is dependent upon angiogenesis $(9,10)$, the number of vessels, blood-flow velocity and intratumoral vessel resistance cannot clearly distinguish malignant from benign breast lesions (11).

Contrast-enhanced ultrasound (CEUS) involves an intravascular contrast agent, which makes it possible to assess lesion hemodynamics in more detail. Over the past two decades, many studies have shown that CEUS can significantly improve the accuracy of breast diagnosis compared with conventional US $(12,13)$. However, it remains controversial due to diagnostic criteria varying across regions and the overlap of enhancement patterns in benign and malignant breast lesions, which is the most important barrier to the application of CEUS.

Based on qualitative analyses of 10 enhancement characteristics, our team generated a CEUS prediction model and evaluated its diagnostic performance. Our initial multicenter study data had shown the prediction model exhibited excellent sensitivity and negative predictive value (89.4\% \& 88.9\%), which was better than the performance of mammography ( $75 \%$ sensitivity) $(14,15)$. At the same time, we also wanted to explore how patient and lesion characteristics (including patient age, BMI, location and size of breast lesions) may affect the enhancement pattern and the accuracy.

The goal of this study was to understand the limitations of CEUS using data from a larger study population of sufficient quality, and to identify factors that influence its diagnostic power, with an aim to more accurately characterize the value of CEUS for assessing and stratifying the risk of malignancy.

\section{Methods}

\section{Study subjects}

This was a retrospective analysis of a multicenter study conducted in eight US centers around China. Approvals from the ethics committee of Sichuan Provincial People's Hospital were obtained for this study (No. 2016 14-1). All participants gave written informed consent. They were enrolled between August 2015 and April 2017.

Inclusion criteria were a conventional preoperative US (consisting of grayscale and Doppler US) revealing breast lesions with BI-RADS 4 or 5 on US. In the presence of multiple lesions in a single patient, the largest one was evaluated by CEUS. Exclusion criteria were contraindications to US contrast, such as, allergy, pregnancy or lactation; pulmonary or respiratory diseases; or previous clinical treatments for breast cancer.

The final study cohort included 1023 consecutive female patients with 1023 breast lesions. All of them subsequently underwent surgery or core biopsy (using 16 G needles) (Figure 1).

\section{Conventional and contrast-enhanced imaging protocol}

We used Mylab 90 (Esaote Medical Systems, Genova, Italy), Philips iU22, Philips iU-Elite (Philips, Bothell WA, USA) and GE LOGIQ E9 (GE Health Systems, Wauwatosa WI, USA) devices with LA523 (4-13 MHz), M6-15 (6-15 MHz) and L12-5 (5-12 MHz) linear array transducers for conventional US. CEUS was performed with LA522 (7 MHz), 9L (9 MHz) and L9-3 (3-9 MHz) linear transducers. The contrast agent used was sulfur hexafluoride microbubbles (SonoVue, Bracco, Milan, 


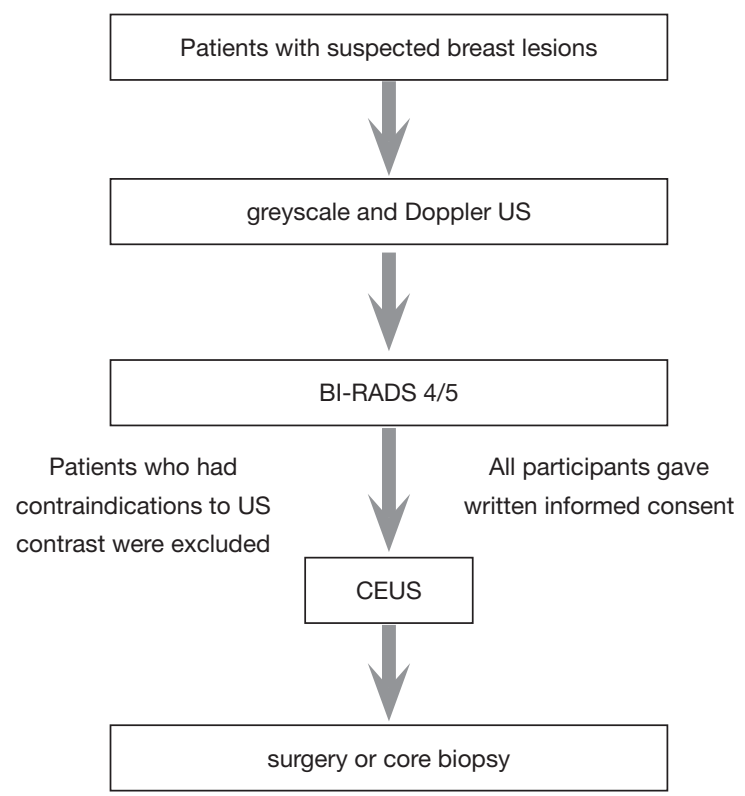

Figure 1 Contrast-enhanced ultrasound flow chart of breast lesions.

Italy), which were reconstituted with $5 \mathrm{~mL}$ of sterile saline. Machine parameters were adjusted with the mechanical index at $\leq 0.13 \mathrm{MHz}$, single focus at the bottom of the image, and the probe stabilized manually with no pressure exerted.

Two US physicians from each center (each with $>15 \mathrm{y}$ of experience with breast US and $2 \mathrm{y}$ of experience with CEUS) were responsible for performing all US and CEUS examinations, during which maximum lesion diameter, distance to the nipple, lesion depth, and distance from the deep edge of the lesion to the pectoralis major were measured and recorded.

During US, operators selected the imaging plane that included the largest area of a visible mass with rich blood supply or any irregularly shaped mass (including its surrounding normal tissue) for CEUS. After a manual bolus injection of $4.8 \mathrm{~mL}$ of contrast via an antecubital vein, the selected plane was again visualized; real-time imaging was recorded for up to $120 \mathrm{~s}$ for further analysis. All static and dynamic images were stored in the US system, and exported in DICOM format.

\section{Image analysis}

Two different physicians from each center (each with $>15$ years of experience with breast US and 2 years of experience with CEUS) evaluated all CEUS images without performing the exam and without knowledge of patients' clinical data, according to the developing CEUS prediction model, as we previously described $(15,16)$. Disagreements were resolved by a third-party appraisal. In brief, this model consists of three malignant $(\mathrm{A} / \mathrm{B} / \mathrm{C}$; Figure 2) and three benign (A/B/C; Figure 3) sub-models.

\section{Pathological data}

Pathological findings of included lesions were collected from each center. The classification of breast lesions was based on the WHO histological classification of tumors of the breast (17).

\section{Statistical analysis}

Statistical analysis was conducted using the SPSS statistical package, version 23.0 for Windows (IBM Corp., Armonk NY, USA). To evaluate the influence of age and body mass index (BMI) on the performance of the CEUS model, patients were subgrouped by WHO standards (for BMI) (18) and previous reports (for age) $(19,20)$. Univariate and multivariate logistic regression analyses were conducted to assess the influence of factors on diagnostic performance of the CEUS model. Receiver operating characteristic (ROC) curves were used to find the optimal cutoff value for distance from the lesion's deep edge to the pectoralis major. An independent-samples $t$-test was applied to compare age, maximum lesion diameter, and distance from the lesion's deep edge to the pectoralis major in malignant and benign lesions. The null hypothesis was rejected at a level of $5 \%(\mathrm{P}<0.05)$.

\section{Results}

\section{CEUS model diagnoses of different pathological breast lesions}

Patients' clinicopathological data are outlined in Table 1. CEUS model diagnoses of pathologically different breast lesions are shown in Table 2. Lesions that met any of the malignant or benign submodel characteristics were defined as malignant or benign respectively; lesions meeting both benign and malignant submodels were defined as malignant.

\section{Factors influencing the performance of the CEUS model}

Univariate analysis of age, BMI, maximum lesion diameter, 

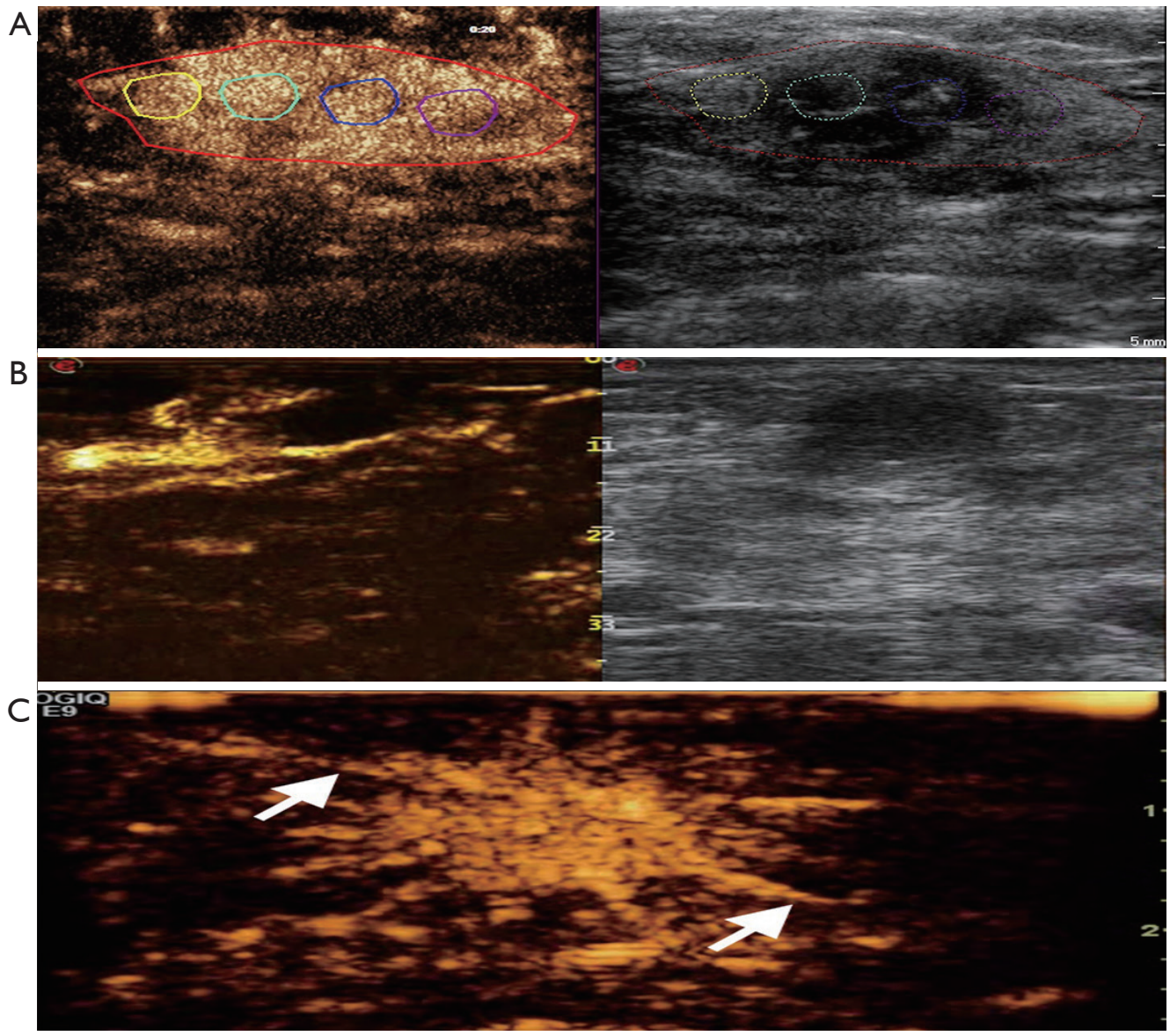

Figure 2 CEUS malignancy submodels for breast lesions. (A) Hyperenhancement with enlarged range, with or without irregular shape; (B) hyper centripetal enhancement with perfusion defect, with or without enlarged range; (C) rapid or synchronous wash-in with hyper- or iso-enhancement, shows penetrating vessels (white arrows) or a crab claw-like pattern, with or without perfusion defect.

distance to the nipple, lesion depth, and distance from the lesion's deep edge to the pectoralis major showed that only age, maximum lesion diameter, and distance from the lesion's deep edge to the pectoralis major significantly affected the CEUS model's performance. Diagnostic accuracy was better for patients $>35 \mathrm{y}$ than for younger patients $(\mathrm{P}=0.005)$. In multivariate regression analysis, age, maximum lesion diameter, and distance from the lesion's deep edge to the pectoralis major remained as independent influence factors for the CEUS model (Table 3).

\section{Influence of maximum lesion diameter and distance from the lesion's deep edge to the pectoralis major on the performance of the CEUS model}

We used stratification analysis to evaluate the influence of maximum lesion diameter and distance from the lesion's deep edge to the pectoralis major on the CEUS model. Patients were subgrouped by maximum lesion diameter according to previous reports (21).

Data revealed that the model was more accurate for lesions with maximum lesion diameter $>20 \mathrm{~mm}$ than for those with maximum lesion diameter $<10 \mathrm{~mm}$ (OR: 1.61); and for lesions with distance from the lesion's deep edge to the pectoralis major $\leq 3.05 \mathrm{~mm}$ that those with distance from the lesion's deep edge to the pectoralis major $>3.05 \mathrm{~mm}$ (OR: 0.55; Table 4). There was no significant difference in accuracy between lesions of maximum diameter 10-20 and $<10 \mathrm{~mm}(\mathrm{P}=0.393)$.

\section{Comparison of age, maximum lesion diameter, and distance from the lesion's deep edge to the pectoralis major in malignant and benign lesions}

As age, maximum lesion diameter, and distance from the 


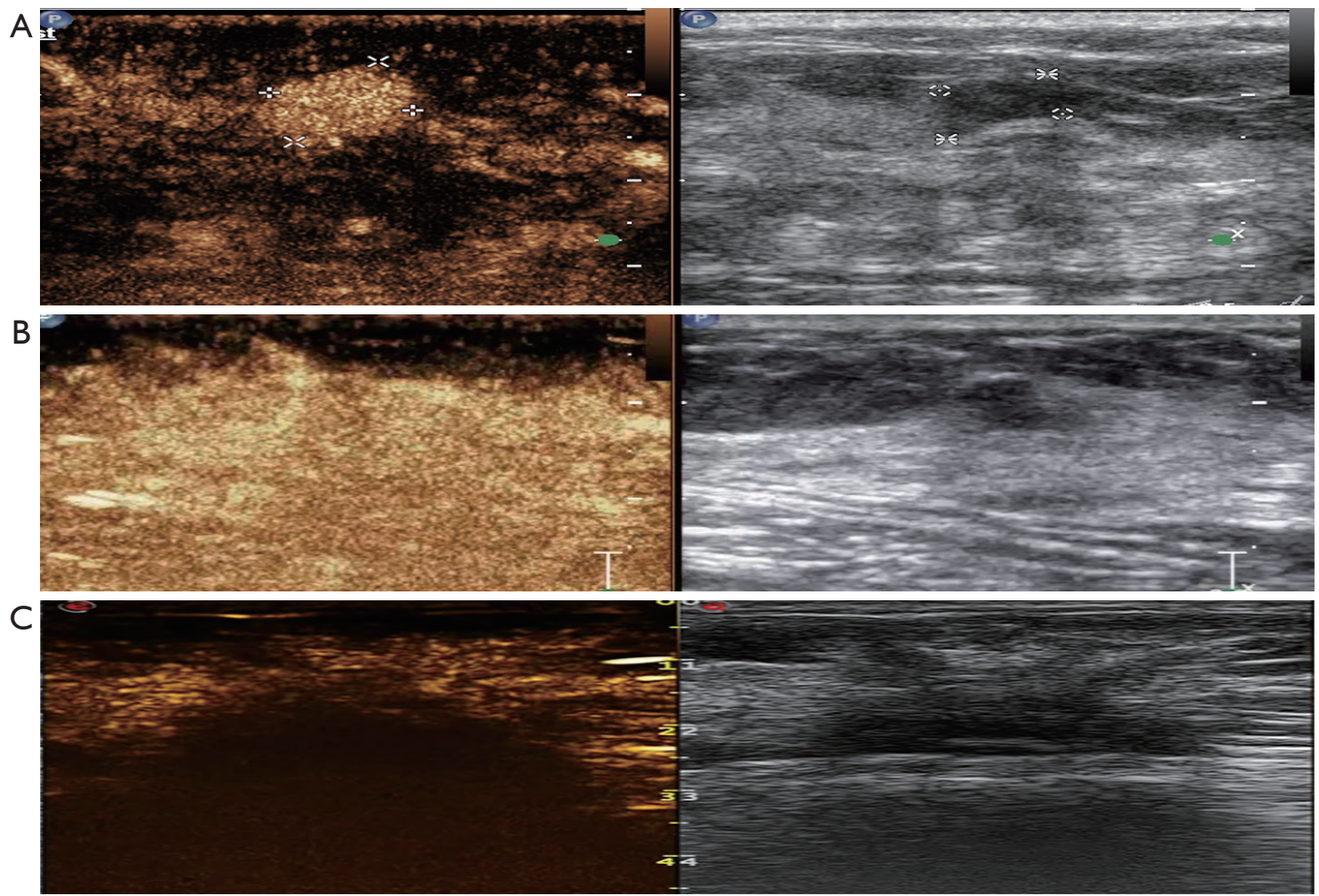

Figure 3 CEUS benign submodels for breast lesions. (A) Rapid wash-in with hyperenhancement, clear margin after enhancement without enlarged size; (B) synchronous or slow wash-in with isoenhancement; margin and shape cannot be distinguished after enhancement; (C) synchronous or slow wash-in with hypoenhancement.

lesion's deep edge to the pectoralis major significantly affected the performance of CEUS model, we compared differences in their effects on malignant and benign lesions. We found that the benign lesion group had significantly $(\mathrm{P}<0.001)$ lower mean maximum lesion diameters and significantly $(\mathrm{P}<0.001)$ higher mean distances from the lesion's deep edge to the pectoralis major than those of the malignant lesion group. The mean age of patients in the benign lesion group was significantly $(\mathrm{P}<0.001)$ younger than that in the malignant lesion group (Table 5).

\section{Discussion}

Our results show that patients' age, maximum lesion diameter, and distance from the lesion's deep edge to the pectoralis major independently influenced the performance of the CEUS model. The model's diagnostic accuracy was significantly better for patients $>35 \mathrm{y}$, for maximum lesion diameter $>20 \mathrm{~mm}$, and for distance from the lesion's deep edge to the pectoralis major $\leq 3.05 \mathrm{~mm}$. CEUS is a promising method for the accurate diagnosis of breast cancer and can optimize BI-RADS categorization (22-25). The results of this study have important implications for physicians performing CEUS for breast lesions.

Patients' age and BMI have been associated with breast cancer risk $(26,27)$. In our study, we addressed the effects of age and BMI on the diagnostic accuracy of CEUS model, and found that age-but not BMI-significantly affected the performance of the current CEUS model. Breast cancer has a significant upward trend in age-standardized incidence rates, but its peak age in Asians is 10-15 y younger than that in Western countries (28). Our study showed our model is more accurate for patients $>35 \mathrm{y}$. Breast lesions are affected by hormone levels and reproductive behavior. Thus, growth patterns of lesions, especially benign lesions, vary greatly among different age groups. In the present study, $42.5 \%(71 / 167)$ of benign lesions in patients $<35 \mathrm{y}$ were misdiagnosed, compared with only $28.4 \%$ (117/412) in women $>35 \mathrm{y}$. Among patients with fibroadenoma, which accounted for $43.9 \%$ of benign lesions, patients $<35 \mathrm{y}$ 
Table 1 Patients' clinicopathological data

\begin{tabular}{|c|c|}
\hline Variables & No. of patients (\%) \\
\hline \multicolumn{2}{|l|}{ Age } \\
\hline Median (range) & $44.71[18-81]$ \\
\hline$\leq 35$ & $203(19.8)$ \\
\hline$>35$ & $820(80.2)$ \\
\hline \multicolumn{2}{|l|}{ BMI } \\
\hline Median (range) & $22.88(14.74-50.78)$ \\
\hline$<25.00 \mathrm{~kg} / \mathrm{m}^{2}$ & $803(78.5)$ \\
\hline$\geq 25.00-29.99 \mathrm{~kg} / \mathrm{m}^{2}$ & $197(19.3)$ \\
\hline$\geq 30.00 \mathrm{~kg} / \mathrm{m}^{2}$ & $23(2.2)$ \\
\hline Maximum lesion diameter & $18.78(4.00-85.00)$ \\
\hline Distance to the nipple & $23.50(0.00-89.00)$ \\
\hline Lesion depth & $6.22(0.00-28.00)$ \\
\hline $\begin{array}{l}\text { Distance from the lesion's deep edge to } \\
\text { the pectoralis major }\end{array}$ & $3.33(0.00-32.00)$ \\
\hline \multicolumn{2}{|l|}{ Histopathology } \\
\hline \multicolumn{2}{|l|}{ Benign lesions ( $n=579)$} \\
\hline Fibroadenoma & 254 (43.9) \\
\hline Fibrocystic mastopathy & $132(22.8)$ \\
\hline Hyperplasia & $72(12.4)$ \\
\hline Granulomatous mastitis & $43(7.4)$ \\
\hline Intraductal papilloma & $42(7.3)$ \\
\hline Complex sclerosing adenosis & $15(2.6)$ \\
\hline Atypical hyperplasia & $11(1.9)$ \\
\hline Others & $10(1.7)$ \\
\hline \multicolumn{2}{|l|}{ Malignant lesions $(n=444)$} \\
\hline IDC & $389(87.6)$ \\
\hline DCIS & $32(7.2)$ \\
\hline Mucinous carcinoma & $11(2.5)$ \\
\hline Others & $12(2.7)$ \\
\hline
\end{tabular}

DCIS, ductal carcinoma in situ; IDC, invasive ductal carcinoma.

had a misdiagnosis rate of $31.1 \%$ (28/90), compared with $20.1 \%(33 / 164)$ in patients $>35 \mathrm{y}$. This phenomenon may be due to the rapid growth of fibroadenoma with active ductal epithelial hyperplasia in young patients, which can be mistaken for sub-model A breast cancer, whereas lesions grow much more slowly and with less neovascularity in patients $>35$ y (29). Those lesions fit the profile of submodel $\mathrm{D}$ and can be correctly diagnosed.

The higher misdiagnosis rate of benign lesions might also be attributed to the significant differences of age, maximum lesion diameter, and distance from the lesion's deep edge to the pectoralis major between malignant and benign lesions. As shown by our study (Table 5), benign lesions occur at a younger age, with lower maximum lesion diameter and higher mean distance from the lesion's deep edge to the pectoralis major, which all poorly affect diagnostic accuracy under the current model. These findings indicate that further study should be conducted to refine and improve the proposed model of CEUS for breast lesions. Adequate consideration of these influencing factors is required to achieve optimal diagnostic power.

Lesion size and depth independently affected the diagnosis accuracy of the CEUS model. Stratification analysis revealed that lesions with maximum lesion diameter $>20 \mathrm{~mm}$ had higher diagnostic accuracy than did those with maximum lesion diameter $<10 \mathrm{~mm}$ (OR: 1.61); There was no significant difference between maximum lesion diameter $10-20$ and $<10 \mathrm{~mm}(\mathrm{P}=0.393)$. Lesion size is closely associated with vessel density, which is essential for the growth and infiltration of breast lesions (30-32). Zhao et al. found that the diagnostic value of CEUS varied for different sizes of lesions (33). Our study showed the specificity of the CEUS model in identifying suspicious lesions with maximum lesion diameter $<10 \mathrm{~mm}$ to be $68.33 \%$. Generally, lesions with maximum lesion diameter $<10 \mathrm{~mm}$ have no typical features, little neovascularization, and their heterogeneity is unclear, whereas larger lesions display more irregular vessel branching with unbalanced spatial distribution and more fine terminal vessels (34). Actually, identifying the intensity or type of enhancement in small lesions was difficult. As Zhang et al. noted (21), subjective judgment played a major role in determining whether there was heterogeneous enhancement in small lesions.

Interestingly, our findings suggest that the efficacy of our CEUS model was more accurate for deep breast lesions than for superficial ones. The influence of distance from the lesion's deep edge to the pectoralis major observed in our study might be generalized to all CEUS diagnoses for breast lesions. Theoretically, the US signal decreases with depth. However, most Asian women have relatively small and dense breasts, with comparatively thin subcutaneous fat layers, posterior adipose tissue, and mammary glands. Therefore, the signal-to-noise ratio of US echo at this depth might not significantly decrease. In addition, lesion 
Table 2 CEUS model diagnoses of different pathological breast lesions

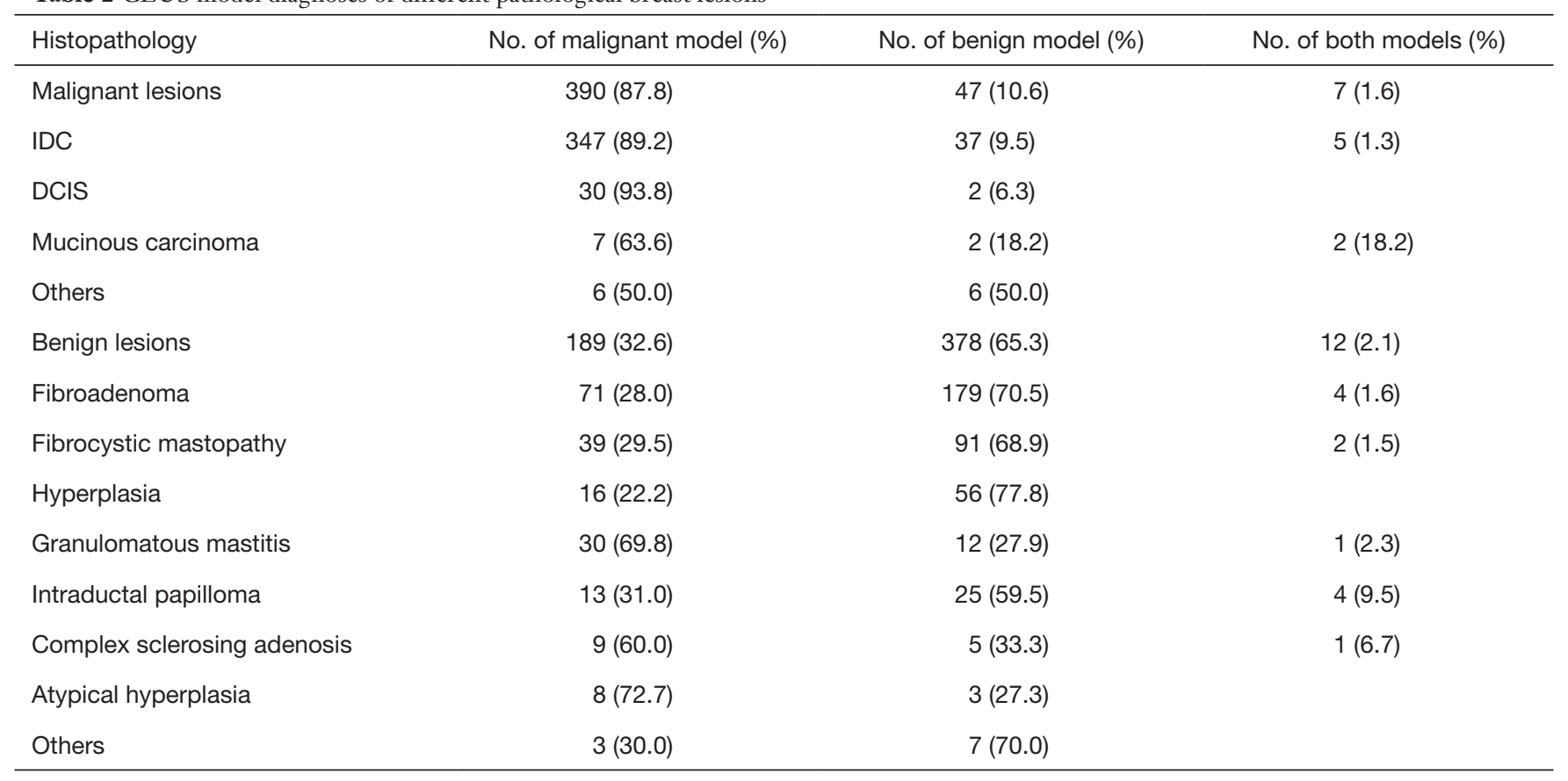

IDC, invasive ductal carcinoma; DCIS, ductal carcinoma in situ.

Table 3 Univariate and multivariate logistic regression analysis of factors that influence the diagnostic performance of the CEUS model

\begin{tabular}{|c|c|c|c|c|c|c|}
\hline Factors & \multicolumn{3}{|c|}{ Univariate } & \multicolumn{3}{|c|}{ Multivariate } \\
\hline Age & & & & 0.67 & $0.47-0.97$ & 0.033 \\
\hline$\leq 35$ & 1 (Reference) & & & & & \\
\hline$>35$ & 0.60 & $0.42-0.86$ & $0.005^{\star}$ & & & \\
\hline$<25.00 \mathrm{~kg} / \mathrm{m}^{2}$ & 1 (Reference) & & & & & \\
\hline$\geq 25.00-29.99 \mathrm{~kg} / \mathrm{m}^{2}$ & 0.57 & $0.24-1.36$ & 0.206 & & & \\
\hline$\geq 30.00 \mathrm{~kg} / \mathrm{m}^{2}$ & 0.69 & $0.28-1.72$ & 0.426 & & & \\
\hline Maximum lesion diameter & 0.97 & $0.96-0.99$ & $0.003^{*}$ & 0.98 & $0.97-0.99$ & 0.038 \\
\hline $\begin{array}{l}\text { Distance from the lesion's deep } \\
\text { edge to the pectoralis major }\end{array}$ & 1.09 & $1.04-1.13$ & $0.000^{*}$ & 1.09 & $1.05-1.14$ & 0.000 \\
\hline Constant & & & & 0.52 & & 0.010 \\
\hline
\end{tabular}

${ }^{*}$, P values $<0.05$. CEUS, contrast-enhanced ultrasound. 
Table 4 Diagnostic performance of the CEUS model with different maximum lesion diameters and different distances from the lesion's deep edge to the pectoralis major

\begin{tabular}{|c|c|c|c|c|c|}
\hline Factors & $\mathrm{n}$ & Sensitivity & Specificity & OR & $\mathrm{P}$ \\
\hline \multicolumn{6}{|c|}{ Maximum lesion diameter } \\
\hline$<10 \mathrm{~mm}$ & 211 & $87.10(69.24-95.78)$ & $68.33(60.93-74.94)$ & 1 (Reference) & \\
\hline $10-20 \mathrm{~mm}$ & 420 & $86.59(80.18-91.22)$ & $66.41(60.22-72.10)$ & $1.18(0.81-1.70)$ & 0.393 \\
\hline$>20 \mathrm{~mm}$ & 392 & $91.57(87.22-94.58)$ & $59.44(50.90-67.47)$ & $1.61(1.10-2.37)$ & $0.016^{\star}$ \\
\hline$>3.05 \mathrm{~mm}$ & 404 & $83.62(75.35-89.61)$ & $63.19(57.31-68.72)$ & 1 (Reference) & \\
\hline$\leq 3.05 \mathrm{~mm}$ & 619 & $91.46(87.76-94.15)$ & $67.35(61.59-72.64)$ & $1.81(1.35-2.41)$ & $<0.001^{*}$ \\
\hline
\end{tabular}

$95 \% \mathrm{Cl}$ shown in parentheses. *, $\mathrm{P}$ values $<0.05$. CEUS, contrast-enhanced ultrasound.

Table 5 Comparisons of patient age, maximum lesion diameter, and distance from the lesion's deep edge to the pectoralis major in malignant and benign lesions

\begin{tabular}{lcc}
\hline Factors & Benign lesions & Malignant lesions \\
\hline Age & $41.24 \pm 9.73$ & $49.50 \pm 10.65$ \\
Maximum lesion diameter & $14.78 \pm 8.56$ & $23.35 \pm 10.49$ \\
Distance from the lesion's deep edge to the pectoralis major & $3.75 \pm 3.01$ & $2.41 \pm 2.69<0.001^{*}$ \\
\hline
\end{tabular}

*, $P$ values $<0.05$.

size and depth are inseparable factors; when the lesion is larger or grows longitudinally, distance from the lesion's deep edge to the pectoralis major is usually shorter. Also, although external force from probe manipulation could also affect the display of microcirculation perfusion, the influence of probe pressure tends to decrease for deeper lesions. This finding suggests that the CEUS model could supplement elastography to assess deep breast lesions and provide greater diagnostic confidence in the context of our specific study population (Chinese women) $(35,36)$.

Although this retrospective study has evaluated factors that most commonly affect conventional US or CEUS findings, other factors, such as breast thickness, were not considered, and may require further study. Additionally, because of the multicenter design, different imaging systems were used among the centers.

In conclusion, although the application of this current CEUS model to breast lesions is clinically promising, it still has its limitations. Age, maximum lesion diameter, and distance from the lesion's deep edge to the pectoralis major significantly affect diagnostic performance and should be considered for the most accurate diagnostic findings with the CEUS model. Further studies are necessary to refine the prediction model and improve accuracy in order to expand the application of CEUS for breast lesions.

Ultimately, the physician and patient have the responsibility to jointly explore and select the most appropriate ultrasound technologies among the available alternatives.

\section{Acknowledgments}

We thank Libby Cone, MD, MA, of Liwen Bianji, Edanz Group China (www.liwenbianji.cn/ac), for editing the English text of drafts of this manuscript.

Funding: The work was sponsored by Sichuan Provincial People's Hospital (\#2017HX001), Fujian Provincial Health Technology Project (\#2015-1-11), and Science and Technology Program of Fujian Province, China (\#2018Y2003).

\section{Footnote}

Conflicts of Interest: The authors have no conflicts of interest 
to declare.

Ethical Statement: The authors are accountable for all aspects of the work in ensuring that questions related to the accuracy or integrity of any part of the work are appropriately investigated and resolved. Approvals from the ethics committee of Sichuan Provincial People's Hospital were obtained for this study (No. 2016 14-1). All participants gave written informed consent.

\section{References}

1. Bray F, Ferlay J, Soerjomataram I, et al. Global cancer statistics 2018: GLOBOCAN estimates of incidence and mortality worldwide for 36 cancers in 185 countries. CA Cancer J Clin 2018;68:394-424.

2. Caplan L. Delay in breast cancer: implications for stage at diagnosis and survival. Front Public Health 2014;2:87.

3. Ohuchi N, Suzuki A, Sobue T, et al. Sensitivity and specificity of mammography and adjunctive ultrasonography to screen for breast cancer in the Japan Strategic Anti-cancer Randomized Trial (J-START): a randomised controlled trial. Lancet 2016;387:341-8.

4. Rajaram N, Mariapun S, Eriksson M, et al. Differences in mammographic density between Asian and Caucasian populations: a comparative analysis. Breast Cancer Res Treat 2017;161:353-62.

5. Radiology ACo, editor. BI-RADS: ultrasound. In: Breast Imaging Reporting and Data System: BI-RADS Atlas (4th edn). Reston. VA: American College of Radiology; 2003.

6. Bevers TB, Anderson BO, Ermelinda B, et al. NCCN clinical practice guidelines in oncology: breast cancer screening and diagnosis. J Natl Compr Canc Netw 2009;7:1060-96.

7. Klimberg VS, Rivere A. Ultrasound image-guided core biopsy of the breast. Chin Clin Oncol 2016;5:33.

8. Heinig J, Witteler R, Schmitz R, et al. Accuracy of classification of breast ultrasound findings based on criteria used for BI-RADS. Ultrasound Obstet Gynecol 2008;32:573-8.

9. Li YJ, Wen G, Yang L, et al. Heterogeneity of angioarchitecture and their hemodynamic changes in benign and malignant breast tumors. Zhonghua Zhong Liu Za Zhi 2009;31:24-7.

10. Sun C, Li J, Wang B, et al. Tumor angiogenesis and bone metastasis - Correlation in invasive breast carcinoma. J Immunol Methods 2018;452:46-52.

11. Svensson WE, Pandian AJ, Hashimoto H, et al. The use of breast ultrasound color Doppler vascular pattern morphology improves diagnostic sensitivity with minimal change in specificity. Ultraschall Med 2010;31:466-74.

12. Xiao X, Ou B, Yang H, et al. Breast contrast-enhanced ultrasound: is a scoring system feasible? A preliminary study in China. PLoS One 2014;9:e105517.

13. Guo R, Lu G, Qin B, et al. Ultrasound Imaging Technologies for Breast Cancer Detection and Management: A Review. Ultrasound Med Biol 2018;44:37-70.

14. Zhang Y, Ren H. Meta-analysis of diagnostic accuracy of magnetic resonance imaging and mammography for breast cancer. J Cancer Res Ther 2017;13:862.

15. Tang L, Chen Y, Du Z, et al. A multicenter study of a contrast-enhanced ultrasound diagnostic classification of breast lesions. Cancer Manag Res 2019;11:2163-70.

16. Luo J, Chen JD, Chen Q, et al. Predictive model for contrast-enhanced ultrasound of the breast: Is it feasible in malignant risk assessment of breast imaging reporting and data system 4 lesions? World J Radiol 2016;8:600-9.

17. Lakhani SR, Ellis IO, Schnitt SJ, et al. WHO Classification of Tumours of the Breast (IARC WHO Classification of Tumours). Lyon: IARC Press; 2012.

18. Anuurad E, Shiwaku K, Nogi A, et al. The New BMI Criteria for Asians by the Regional Office for the Western Pacific Region of WHO are Suitable for Screening of Overweight to Prevent Metabolic Syndrome in Elder Japanese Workers. J Occup Health 2003;45:335-43.

19. Darwish AD, Helal AM, El-Din NHA, et al. Breast cancer in women aging 35 years old and younger: The Egyptian National Cancer Institute (NCI) experience. Breast 2017;31:1-8.

20. Cancello G, Maisonneuve P, Rotmensz N, et al. Prognosis and adjuvant treatment effects in selected breast cancer subtypes of very young women ( $<35$ years) with operable breast cancer. Ann Oncol 2010;21:1974-81.

21. Zhang JX, Cai LS, Chen L, et al. CEUS helps to rerate small breast tumors of BI-RADS category 3 and category 4 . Biomed Res Int 2014;2014:572532.

22. Liu GF, Wang ZQ, Zhang SH, et al. Diagnostic and prognostic values of contrast-enhanced ultrasound combined with diffusion-weighted magnetic resonance imaging in different subtypes of breast cancer. Int J Mol Med 2018;42:105-14.

23. Yuan Z, Quan J, Yunxiao Z, et al. Diagnostic value of contrast-enhanced ultrasound parametric imaging in breast tumors. Journal of Breast Cancer 2013;16:208-13.

24. Liu H, Jiang YX, Liu JB, et al. Evaluation of breast lesions with contrast-enhanced ultrasound using the 
microvascular imaging technique: initial observations. Breast 2008; 17:532-9.

25. Zhao H, Xu R, Ouyang Q, et al. Contrast-enhanced ultrasound is helpful in the differentiation of malignant and benign breast lesions. Eur J Radiol 2010;73:288-93.

26. Guo Y, Warren Andersen S, Shu XO, et al. Genetically Predicted Body Mass Index and Breast Cancer Risk: Mendelian Randomization Analyses of Data from 145,000 Women of European Descent. PLoS Med 2016;13:e1002105.

27. Picon-Ruiz M, Morata-Tarifa C, Valle-Goffin JJ, et al. Obesity and adverse breast cancer risk and outcome: Mechanistic insights and strategies for intervention. CA Cancer J Clin 2017;67:378-97.

28. Chen W, Zheng R, Baade PD, et al. Cancer statistics in China, 2015. CA Cancer J Clin 2016;66:115-32.

29. Kim SJ, Park YM, Jung SJ, et al. Sonographic appearances of juvenile fibroadenoma of the breast. J Ultrasound Med 2014;33:1879-84.

30. del Cura JL, Elizagaray E, Zabala R, et al. The use of unenhanced Doppler sonography in the evaluation of solid breast lesions. AJR Am J Roentgenol 2005;184:1788-94.

Cite this article as: Chen Y, Tang L, Du Z, Zhong Z, Luo J, Yang L, Shen R, Cheng Y, Zhang Z, Han E, Lv Z, Yuan L, Yang Y, Cheng Y, Yang L, Wang S, Bai B, Chen Q. Factors influencing the performance of a diagnostic model including contrast-enhanced ultrasound in 1023 breast lesions: comparison with histopathology. Ann Transl Med 2019;7(22):647. doi: 10.21037/atm.2019.10.83
31. Lai HW, Chen DR, Wu YC, et al. Comparison of the Diagnostic Accuracy of Magnetic Resonance Imaging with Sonography in the Prediction of Breast Cancer Tumor Size: A Concordance Analysis with Histopathologically Determined Tumor Size. Ann Surg Oncol 2015;22:3816-23.

32. Goubran HA, Kotb RR, Stakiw J, et al. Regulation of tumor growth and metastasis: the role of tumor microenvironment. Cancer Growth Metastasis 2014;7:9-18.

33. Zhao YX, Liu S, Hu YB, et al. Diagnostic and prognostic values of contrast-enhanced ultrasound in breast cancer: a retrospective study. Onco Targets Ther 2017;10:1123-9.

34. Balleyguier C, Opolon P, Mathieu MC, et al. New potential and applications of contrast-enhanced ultrasound of the breast: Own investigations and review of the literature. Eur J Radiol 2009;69:14-23.

35. Song G, Jing L, Yan M, et al. Influence of various breast factors on the quality of strain elastograms. J Ultrasound Med 2015;34:395-400.

36. Ciurea AI, Bolboaca SD, Ciortea CA, et al. The influence of technical factors on sonoelastographic assessment of solid breast nodules. Ultraschall Med 2011;32:S27-34. 